\title{
Unpolarized and Helicity Generalized Parton Distributions of the Proton within Lattice QCD
}

\author{
Constantia Alexandrou, ${ }^{1,2}$ Krzysztof Cichy, ${ }^{3}$ Martha Constantinou $\odot,{ }^{4}$ Kyriakos Hadjiyiannakou, ${ }^{1}$ \\ Karl Jansen, ${ }^{5}$ Aurora Scapellato, ${ }^{3}$ and Fernanda Steffens ${ }^{6}$ \\ (Extended Twisted Mass Collaboration) \\ ${ }^{1}$ Department of Physics, University of Cyprus, P.O. Box 20537, 1678 Nicosia, Cyprus \\ ${ }^{2}$ Computation-based Science and Technology Research Center, The Cyprus Institute, 20 Kavafi Street, Nicosia 2121, Cyprus \\ ${ }^{3}$ Faculty of Physics, Adam Mickiewicz University, Uniwersytetu Poznańskiego 2, 61-614 Poznań, Poland \\ ${ }^{4}$ Department of Physics, Temple University, Philadelphia, Pennsylvania 19122-1801, USA \\ ${ }^{5}$ NIC, DESY, Platanenallee 6, D-15738 Zeuthen, Germany \\ ${ }^{6}$ Institut für Strahlen- und Kernphysik, Rheinische Friedrich-Wilhelms-Universität Bonn, Nussallee 14-16, 53115 Bonn, Germany
}

(Received 31 August 2020; accepted 18 November 2020; published 23 December 2020)

\begin{abstract}
We present the first calculation of the $x$ dependence of the proton generalized parton distributions (GPDs) within lattice QCD. Results are obtained for the isovector unpolarized and helicity GPDs. We compute the appropriate matrix elements of fast-moving protons coupled to nonlocal operators containing a Wilson line. We present results for proton momenta $0.83,1.25,1.67 \mathrm{GeV}$, and momentum transfer squared $0.69,1.38 \mathrm{GeV}^{2}$. These combinations include cases with zero and nonzero skewness. The calculation is performed using one ensemble of two degenerate mass light quarks, a strange quark, and a charm quark of maximally twisted mass fermions with a clover term. The lattice results are matched to the light-cone GPDs using one-loop perturbation theory within the framework of large momentum effective theory. The final GPDs are given in the $\overline{\mathrm{MS}}$ scheme at a scale of $2 \mathrm{GeV}$.
\end{abstract}

DOI: 10.1103/PhysRevLett.125.262001

Introduction.-Quantum chromodynamics (QCD) is the fundamental theory describing the strong interactions among quarks and gluons (partons). The strong force is responsible for binding partons into hadrons, such as the proton, that makes the bulk of the visible matter in the universe. Studying how the properties of protons emerge from the underlying constituents and their interactions has been an important experimental and theoretical endeavor since the mid-20th century. These studies led to the realization that high-energy scattering processes can be factorized into perturbative and nonperturbative parts. The latter includes information about the parton structure of the proton [1]. This resulted in the introduction of a complete set of key quantities, namely the parton distribution functions (PDFs) [1], generalized parton distributions (GPDs) [2-4], and transverse momentum dependent distributions (TMDs) $[5,6]$. These describe the nonperturbative dynamics of the proton, and in general

Published by the American Physical Society under the terms of the Creative Commons Attribution 4.0 International license. Further distribution of this work must maintain attribution to the author(s) and the published article's title, journal citation, and DOI. Funded by SCOAP. hadrons, in terms of their constituent quarks and gluons [7].

There are two unpolarized GPDs, $H^{q}(x, \xi, t)$ and $E^{q}(x, \xi, t)$, and two helicity GPDs, $\tilde{H}^{q}(x, \xi, t)$ and $\tilde{E}^{q}(x, \xi, t)$. The superscript $q$ refers to a given quark flavor, and here we study the isovector combination $u-d$. GPDs are functions not only of the longitudinal momentum fraction $x(0 \leq x \leq 1)$ carried by the partons, but also of the skewness $\xi \equiv-\Delta^{+} / 2 P^{+}$and the momentum transfer squared, $t \equiv \Delta^{2} . \Delta^{+}$and $P^{+}$are the plus component of the momentum transfer and the average proton momentum, respectively. Two kinematical regions arise based on the values of $\xi$ and $x$ : the so-called Dokshitzer-Gribov-LipatovAltarelli-Parisi (DGLAP) region [8-11] defined for $x>|\xi|$, and the Efremov-Radyushkin-Brodsky-Lepage (ERBL) $[12,13]$ region for $x<|\xi|$. Physical content can be attributed to each region [14] using light-cone coordinates and the light-cone gauge. In the positive- (negative-) $x$ DGLAP region, the GPDs correspond to the amplitude of removing a quark (antiquark) of momentum $k$ from the hadron, and then inserting it back with momentum $k+\Delta$. In the ERBL region, the GPD is the amplitude for removing a quark-antiquark pair with momentum $-\Delta$.

While GPDs are multidimensional objects, they lead to simpler quantities when certain limits are taken, or when 
integrating over selected variables. For example, the forward limit of the unpolarized case, $\Delta=0$, gives the quark, $f_{1}(x)=H^{q}(x, 0,0)$, and antiquark PDFs, $\bar{f}_{1}(x)=$ $-H^{q}(-x, 0,0)$. Equivalently, in the helicity case one has $g_{1}(x)=\tilde{H}^{q}(x, 0,0)$ and $\bar{g}_{1}(x)=\tilde{H}^{q}(-x, 0,0)$. Integrating over $x$ for nonzero $\Delta$, GPDs give the usual form factors (FFs). Taking integrals of GPDs over $x$ leads to a tower of Mellin moments that also have a physical interpretation, such as the total angular momentum of quarks using Ji's sum rule [2].

The connection of GPDs with other quantities demonstrates the information they encode, in both coordinate and momentum spaces. GPDs are accessed through deeply virtual Compton scattering (DVCS) and deeply virtual meson production (DVMP) [15]. Despite their importance, it is very difficult to extract them experimentally, even though data are available since the early 2000s. These data are limited, covering a small kinematic region, and are indirectly related to GPDs through the Compton FFs. This poses limitations in their extraction, and the fact that more than one independent measurement is needed to disentangle them [16-19].

Nevertheless, the interest in GPDs is renewed due to the advances both on the experimental and the theoretical side, as well as the expertise gained from recent studies of PDFs. It is, thus, of utmost importance to have ab initio computations of GPDs, that will help map them over different regions of $x$, $\xi$, and $t$. Lattice QCD is the only known formulation that allows a quantitative study of QCD directly using its Lagrangian. Lattice QCD is based on a discretization of Euclidean spacetime and relies on large-scale simulations.

Since parton distributions are light-cone correlation functions [20], it is not straightforward to calculate them using the Euclidean lattice formulation of QCD. The large momentum effective theory (LAMET) proposed by Ji [21] provides a promising theoretical framework to extract lightcone quantities using matrix elements computed in lattice QCD. Within the LAMET [22,23], one can access lightcone quantities via matrix elements of boosted hadrons coupled with nonlocal spatial operators, which are calculable on the lattice, and yield what is referred to as quasidistributions. The first investigations led to encouraging results on the determination of PDFs [24,25]. Since then, the method has been advanced and has attracted a lot of attention, see, e.g., Refs. [26-63], and revitalized other approaches [64-70], as well as given rise to the development and investigation of new ones [71-93] (for recent reviews, see Refs. [94-96]). Recently, a preliminary study of nucleon GPDs was also presented, demonstrating the applicability of the quasidistribution methodology to GPDs [97]. The quasi-GPDs approach has also been studied using the scalar diquark spectator model $[98,99]$.

Extracting GPDs using lattice QCD.-For the calculation of GPDs, we define quasidistributions with boosted proton states and introduce momentum transfer (denoted $Q$ in Euclidean spacetime) between the initial and final states. The matrix element of interest is given by
$h_{\Gamma}\left(z, P_{3}, t, \xi\right) \equiv\left\langle N\left(P_{f}\right)|\bar{\psi}(z) \Gamma W(0, z) \psi(0)| N\left(P_{i}\right)\right\rangle$,

where $\left|N\left(P_{i}\right)\right\rangle\left(\left|N\left(P_{f}\right)\right\rangle\right)$ is the initial (final) state labeled by its momentum, and $t=-Q^{2}$. For simplicity, we drop the index $q$, since in this work we only consider isovector quantities. The boost is in the direction of the Wilson line $(W(0, z)), P_{3}=\left(P_{i 3}+P_{f_{3}}\right) / 2$. Quasi-GPDs depend on the quasiskewness, defined as $\xi=-P_{f_{3}}-P_{i 3} / P_{f_{3}}+$ $P_{i 3}=-\left(Q_{3} / 2 P_{3}\right)$ and equal to the light-cone skewness up to power corrections. The Dirac structure $\Gamma$ defines the type of GPD, and we employ $\gamma_{0}$ and $\gamma_{5} \gamma_{3}$ for the unpolarized and helicity GPDs, respectively [100].

Another aspect of the calculation is the renormalization, as the divergences with respect to the regulator must be removed prior to applying Eq. (4). We adopt [101] the nonperturbative renormalization scheme of Refs. [29,30], and refined in Ref. [47]. This procedure removes all divergences, including the power-law divergence with respect to the ultraviolet cutoff. The renormalization functions, $Z_{\Gamma}$, are obtained nonperturbatively by imposing Regularization-Independent (RI) type [102] renormalization conditions, given in Eq. (S10). In a nutshell, the final values of $Z_{\Gamma}$ are obtained at each value of $z$ separately, at a chosen RI scale $\left(a \mu_{0}\right)^{2}$. For each value of $z$ at a given $\mu_{0}$, we take the chiral limit using a linear fit in $m_{\pi}^{2}$. As described in the Supplemental Material [103], the available matching equations [104] require that the quasi-GPDs are in the RI scheme. Therefore, we renormalize the matrix elements using the estimates for $Z_{\Gamma}$ in the RI scheme at a given scale, $\mu_{0}$, chosen to be $\left(a \mu_{0}\right)^{2} \approx 1.17$. This scale enters the matching kernel, which converts the quasi-GPDs to lightcone GPDs. The latter are always given in the $\overline{\mathrm{MS}}$ scheme at $2 \mathrm{GeV}$, regardless of the scheme used for quasi-GPDs. Within this work, we explored a few values of the scale within the range $\left(a \mu_{0}\right)^{2} \in[1-5]$. We find that the dependence on $\left(a \mu_{0}\right)^{2}$ is within the reported uncertainties.

The renormalized matrix elements are decomposed into the form factors $\left\{F_{H}, F_{E}\right\}$ and $\left\{F_{\tilde{H}}, F_{\tilde{E}}\right\}$, for the unpolarized and helicity case, respectively. The decomposition is based on continuum parametrizations, which in Euclidean space take the form

$$
\begin{aligned}
\left\langle N\left(P_{f}\right)\left|\mathcal{O}_{\gamma_{\mu}}(z)\right| N\left(P_{i}\right)\right\rangle= & \left\langle\left\langle\gamma_{\mu}\right\rangle\right\rangle F_{H}\left(z, P_{3}, t, \xi\right) \\
& -i \frac{\left\langle\left\langle\sigma_{\rho \mu}\right\rangle Q_{\rho}\right.}{2 m} F_{E}\left(z, P_{3}, t, \xi\right), \\
\left\langle N\left(P_{f}\right)\left|\mathcal{O}_{\gamma_{\mu} \gamma_{5}}(z)\right| N\left(P_{i}\right)\right\rangle= & \left\langle\left\langle\gamma_{\mu} \gamma_{5}\right\rangle\right\rangle F_{\tilde{H}}\left(z, P_{3}, t, \xi\right) \\
& -i\left\langle\left\langle\gamma_{5}\right\rangle\right\rangle \frac{Q_{\mu}}{2 m} F_{\tilde{E}}\left(z, P_{3}, t, \xi\right),
\end{aligned}
$$

where $Q \equiv P_{f}-P_{i}$, and $m$ is the proton mass. $\mathcal{O}_{\Gamma}(z) \equiv$ $\bar{\psi}(z) \Gamma W(0, z) \psi(0)$ and $\langle\langle\Gamma\rangle\rangle \equiv \bar{u}_{N}\left(P_{f}, s^{\prime}\right) \Gamma u_{N}\left(P_{i}, s\right)$ with $u_{N}$ the proton spinors.

The matrix elements $h_{\Gamma}\left(z, P_{3}, t, \xi\right)$ depend on $z$, which varies from zero up to the half of the spatial extent $L$ of the 
lattice. One way to reconstruct the $x$ dependence of the GPDs is via a standard Fourier transform, e.g., we define the quasi- $H$ GPD as $H_{q}$ :

$H_{q}\left(x, t, \xi, \mu_{0}, P_{3}\right)=\int \frac{d z}{4 \pi} e^{-i x P_{3} z} F_{H}\left(z, P_{3}, t, \xi, \mu_{0}\right)$.

This simple Fourier transform suffers from an ill-defined inverse problem [81]. One alternative reconstruction technique that we adopt here is the Backus-Gilbert (BG) method [106] that leads to a uniquely reconstructed quasidistribution from the available set of matrix elements. More details can be found in the Supplemental Material.

The matching formula is available to one-loop level in perturbation theory, for general skewness [104,105]. In fact, in the limit of $\xi \rightarrow 0$, one recovers the matching equations for quasi-PDFs. Furthermore, the matching kernels of $H$ and $E$ GPDs are the same [104]. We provide details on the matching in the Supplemental Material.

Numerical techniques.-For this calculation, we employ an ensemble with two light quarks, a strange quark, and a charm quark $\left(N_{f}=2+1+1\right)$ using the twisted mass formulation [107,108] with clover improvement [109], generated by the Extended Twisted Mass Collaboration (ETMC) [110]. The ensemble has a spatial (temporal) extent of $3 \mathrm{fm}(6 \mathrm{fm})\left(32^{3} \times 64\right)$, a lattice spacing of $0.093 \mathrm{fm}$ and pion mass of about $260 \mathrm{MeV}$. For the isovector combination $u$ - $d$, we need to evaluate only the connected diagram (see Fig. S1).

To increase the signal-to-noise ratio, we use momentum smearing [111], which has been very successful in the calculation of matrix elements of nonlocal operators with boosted hadrons $[27,38,47,110]$. We find that momentum smearing decreases the gauge noise of the real (imaginary) part a factor of 4 to 5 (2 to 3) (see, e.g., Fig. S2). To further suppress statistical uncertainties, we apply stout smearing [112] to the links of the operator. The effectiveness of the stout smearing in proton matrix elements was demonstrated in Refs. $[113,114]$. While the stout smearing changes the matrix elements, it also alters $Z_{\Gamma}$, and the renormalized matrix elements are independent of the stout smearing.

Ensuring ground-state dominance in $h_{\Gamma}$ is essential and is controlled by the time separation between the source (initial state) and the sink (final state). This separation, $t_{s}$, needs to be large in order to suppress excited-states contributions to the matrix elements. We construct a suitable ratio of two- and three-point functions [see Eq. (S4)], to cancel out unknown overlap factors. Multiple ratios are obtained, for each operator insertion time $t_{\text {ins }}=1, \ldots, t_{s}-a$ (assuming the source time is zero). Ground-state dominance is established when the ratio becomes time independent for values of $t_{\text {ins }}$ (plateau region) that are far away enough from the source and the sink [see Eq. (S5)]. The matrix elements $h_{\Gamma}\left(z, P_{3}, t\right)$ are extracted from a constant fit within the plateau region. Here, we choose $t_{s}=1.12 \mathrm{fm}$ [47], and use the sequential method at fixed $t_{s}$ value.

The most common definition of GPDs is in the Breit frame, in which the momentum transfer $Q$ is equally shared between the initial and final states. This has important implications for the computational cost of extracting $h_{\Gamma}\left(z, P_{3}, t, \xi\right)$ as compared to the usual FFs. For different momentum transfers, both the source and the sink momenta change, requiring separate inversions for each value of $Q$. The statistics used for the results presented in this work are given in Tables SI-SII. We note that, for the largest value of proton momentum, $P_{3}=1.67 \mathrm{GeV}$, the number of measurements required to reach sufficient accuracy is 112192 . The Supplemental Material contains more information on the technical aspects and includes Refs [115-124].

Results for the matrix elements $h_{\Gamma}$.-The renormalized matrix elements are decomposed into $F_{H}, F_{E}, F_{\tilde{H}}$, and $F_{\tilde{E}}$ using Eqs. (2) and (3). To disentangle $F_{H}$ and $F_{E}$, we use $h_{\gamma^{0}}\left(z, P_{3}, t, \xi\right)$ projected with the unpolarized projector, $\mathcal{P}_{0} \equiv\left(1+\gamma^{0}\right) / 4$ and the polarized projector, $\mathcal{P}_{\kappa} \equiv\left(1+\gamma^{0}\right) i \gamma^{5} \gamma^{\kappa} / 4$. For the helicity matrix element, $h_{\gamma^{3} \gamma^{5}}\left(z, P_{3}, t, \xi\right)$, we use the polarized projector, $\mathcal{P}_{\kappa}$, where both $\kappa=3$ and $\kappa \neq 3$ are necessary to disentangle $F_{\tilde{H}}$ and $F_{\tilde{E}}$. We note that for zero skewness, only $\kappa=3$ leads to a nonzero matrix element for the axial vector operator, which is related to $F_{\tilde{H}}$. Thus, for $\xi=0$ we cannot access the $\tilde{E}$ GPD. In fact, the inaccessibility of $\tilde{E}$ GPD is a general feature due to its vanishing kinematic factor at $\xi=0$, and is not related to the choice of the projector.

For the largest momentum, $P_{3}=1.67 \mathrm{GeV}$, we find similar magnitude contributions from both projectors $\mathcal{P}_{0}$ and $\mathcal{P}_{1}$. These matrix elements are combined to solve a system of linear equations to extract $F_{H}$ and $F_{E}$. Because of its kinematic coefficient, $F_{E}$ has, in general, larger errors than those for $F_{H}$. We find that the momentum dependence changes based on the values of $z$, and on the quantity under study. This momentum dependence propagates in a nontrivial way to the final $H$ and $E$ GPDs, as one has to reconstruct the quasi-GPDs in momentum space, and then, apply the appropriate matching formula, which depends on the momentum $P_{3}$. The matrix element $h_{\gamma^{5} \gamma^{3}}$ at zero skewness leads directly to $F_{\tilde{H}}$, as the kinematic factor of $\tilde{E}$ is zero. More details and plots can be found in the Supplemental Material.

Results on the GPDs.-The $P_{3}$ convergence of the GPDs is of particular interest, as the matching kernel is only known to one-loop level. For $H$ GPD and $\tilde{H}$ GPD at $\xi=0$, we find that the momentum dependence is small and within the reported uncertainties. Convergence is also observed for $E$ GPD for the two highest momenta and the region $x>0$. We note that the statistical errors on $E$ GPD are larger than those of the $H$ GPD, a feature already observed in $F_{E}$. We refer the reader to the Supplemental Material for more details.

Our final results for $P_{3}=1.67 \mathrm{GeV}, t=-0.69 \mathrm{GeV}^{2}$, and zero skewness are shown in Figs. 1 and 2 for the 


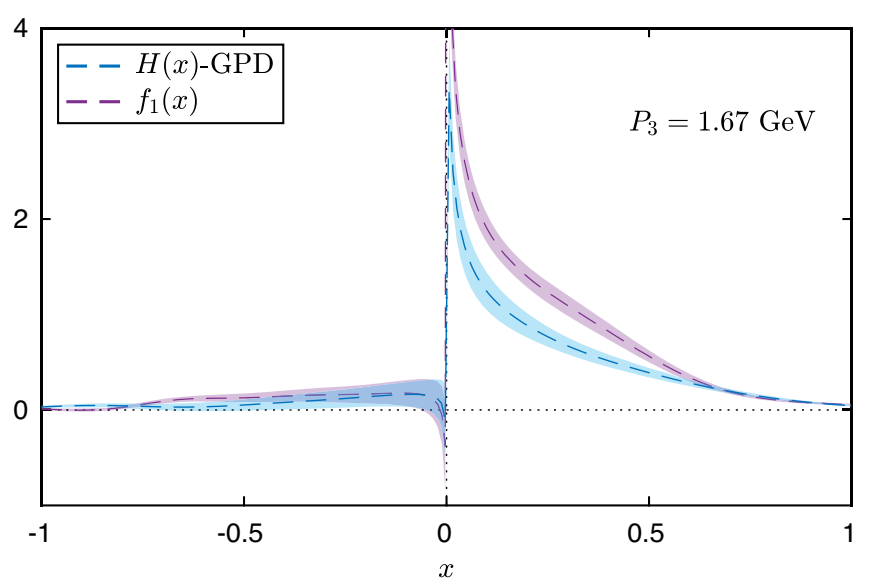

FIG. 1. $\quad H$ GPD (blue band) and unpolarized PDF (violet band) for $P_{3}=1.67 \mathrm{GeV}$ and zero skewness.

unpolarized and helicity GPDs, respectively. For each case, we compare the GPDs with the corresponding PDFs, that is $f_{1}(x)$ for the unpolarized, and $g_{1}(x)$ for the helicity. We observe that the GPDs are suppressed in magnitude as compared to their respective PDFs for all values of $x \lesssim 0.7$. In fact, $\tilde{H}$ GPD has a steeper slope at small $x$ values. The smaller magnitude of the GPDs is a feature also observed in the standard FFs, which decay with increasing $-t$. For the large- $x$ region, both distributions decay to zero in the same way. The large- $x$ behavior of the unpolarized GPD is in agreement with the power counting analysis of Ref. [125]. For the antiquark region, we find that the GPDs are compatible with the corresponding PDFs. We note that the statistical uncertainties of GPDs are similar to the PDFs, allowing for such qualitative comparison.

The extraction of the GPDs for $\xi \neq 0$ differs from the one for $\xi=0$, as a different matching kernel is required. Also, unlike the $\xi=0$ case, both helicity GPDs contribute to the matrix element, and therefore a decomposition is required. The comparison between the zero and nonzero skewness is shown in Figs. 3 and 4 , for $P_{3}=1.25 \mathrm{GeV}$. The main feature of the GPDs at $\xi \neq 0$ is that an ERBL region $(|x|<1 / 3$ in our case) appears, differentiating it from the DGLAP region $(|x|>1 / 3)$. The behavior of the GPDs as a function of $t$ for a fixed $x$ is as expected; increasing $-t$ suppresses the GPDs.

Concluding remarks.-We presented first results on the unpolarized and helicity GPDs for the proton, employing the quasidistribution approach, which has been very successful for the extraction of PDFs within lattice QCD. In the case of GPDs, a nonzero momentum is transferred between boosted initial and final states. The lattice QCD data were renormalized nonperturbatively, and the Backus-Gilbert method was used to extract the $x$ dependence of quasi-GPDs. Applying matching to the latter within the LAMET approach yielded the light-cone GPDs in the $\overline{\mathrm{MS}}$ scheme at $2 \mathrm{GeV}$.

The momentum dependence of GPDs for $P_{3}=0.83$, $1.25,1.67 \mathrm{GeV}$ at fixed $t=-0.69 \mathrm{GeV}^{2}$ (Figs. S8, S9 of

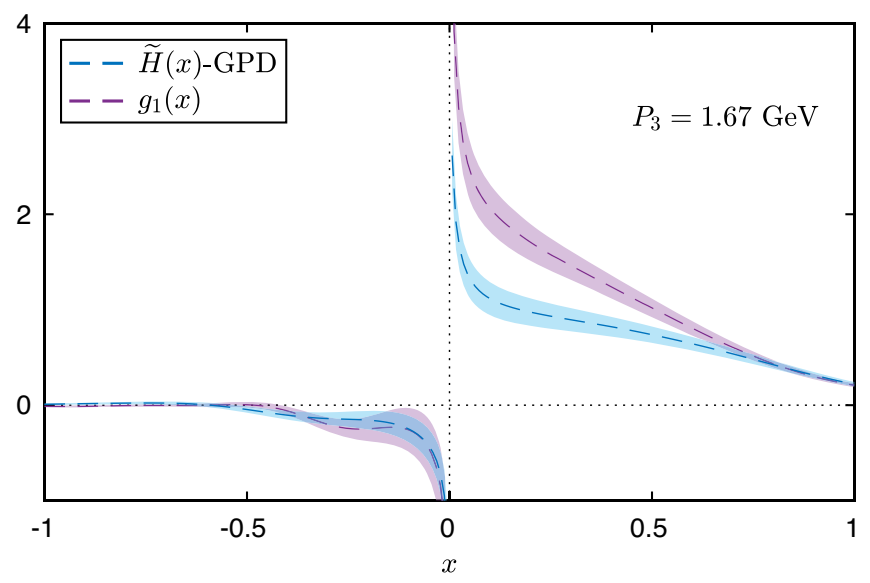

FIG. 2. $\tilde{H}$ GPD (blue band) and helicity PDF (violet band) for $P_{3}=1.67 \mathrm{GeV}$ and zero skewness.

the Supplemental Material) indicates convergence between the largest two momenta. Our final results, given in Figs. 1 and 2 at zero skewness and Figs. 3 and 4 at nonzero skewness, are reassuring, as with increasing $-t$, the magnitude of GPDs is suppressed. With our calculation, we demonstrate that extracting GPDs with controlled statistical uncertainties is feasible. Their accuracy permits qualitative comparison with their corresponding PDFs.

In the near future, we will investigate systematic uncertainties, as studied for PDFs [47]. The pion mass dependence will also be studied using an ensemble with quark masses fixed to their physical values. In a follow-up calculation, we will also explore the transversity GPD, for which there are two additional form factors, leading to a more evolved decomposition. This makes the disentanglement of the transversity GPDs more challenging.

The current work demonstrates the feasibility of the quasidistributions approach for GPDs using computational

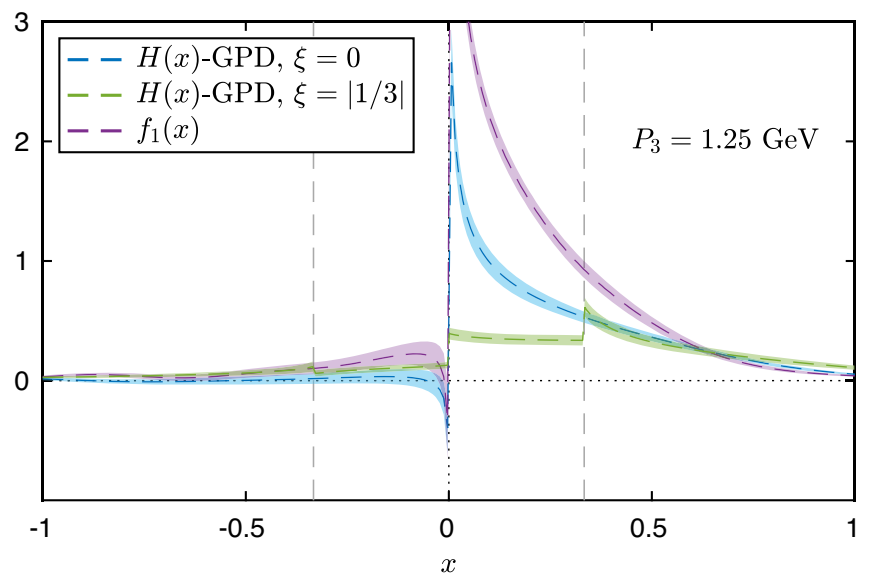

FIG. 3. $\quad H$ GPD for $\xi=0$ (blue band) and $\xi=|1 / 3|$ (green band), as well as the unpolarized PDF (violet band) for $P_{3}=1.25 \mathrm{GeV}$. The area between the vertical dashed lines is the ERBL region. 


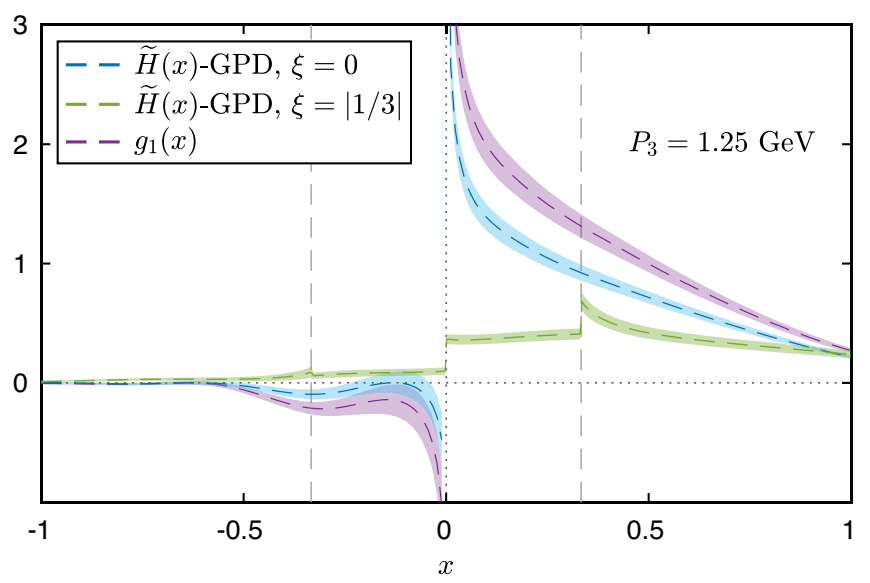

FIG. 4. $\quad \tilde{H}$ GPD for $\xi=0$ (blue band) and $\xi=|1 / 3|$ (green band), as well as the helicity PDF (violet band) for $P_{3}=1.25 \mathrm{GeV}$. The area between the vertical dashed lines is the ERBL region.

resources that are within reach. However, there is still a long way, until statistical and systematic uncertainties become under control. Extracting GPDs within the first principles formulation of lattice QCD can potentially be combined with future experimental data within the global fits framework. This direction is very timely, as GPDs are at the heart of planned experiments at JLab [126] and the Electron-Ion Collider (EIC) [127]. Therefore, GPDs are the objects to drive the efforts of the nuclear and hadronic physics communities for the next few decades.

We would like to thank all members of ETMC for their constant and pleasant collaboration. K. C. and A.S. are supported by National Science Centre (Poland) Grant SONATA BIS No. 2016/22/E/ST2/00013. M. C. acknowledges financial support by the U.S. Department of Energy Early Career Award under Grant No. DE-SC0020405. K. $\mathrm{H}$. is supported by the Cyprus Research and Innovation Foundation under Grant No. POST-DOC/0718/0100. F. S. was funded by DFG Project No. 392578569. Partial support is provided by the European Joint Doctorate program STIMULATE of the European Union's Horizon 2020 research and innovation programme under Grant Agreement No. 765048. Computations for this work were carried out in part on facilities of the USQCD Collaboration, which are funded by the Office of Science of the U. S. Department of Energy. This research was supported in part by PLGrid Infrastructure (Prometheus supercomputer at AGH Cyfronet in Cracow). Computations were also partially performed at the Poznan Supercomputing and Networking Center (Eagle supercomputer), the Interdisciplinary Centre for Mathematical and Computational Modelling of the Warsaw University (Okeanos supercomputer), and at the Academic Computer Centre in Gdańsk (Tryton supercomputer). The gauge configurations have been generated by the Extended Twisted Mass Collaboration on the KNL (A2) Partition of Marconi at CINECA, through the Prace project Pra13_3304 "SIMPHYS."

[1] J. C. Collins, D. E. Soper, and G. F. Sterman, Factorization of Hard Processes in QCD, Advanced Series on Directions in High Energy Physics Vol. 5 (World Scientific, Singapore, 1989), pp. 1-91.

[2] X.-D. Ji, Phys. Rev. Lett. 78, 610 (1997).

[3] A. V. Radyushkin, Phys. Lett. B 380, 417 (1996).

[4] D. Mueller, D. Robaschik, B. Geyer, F. M. Dittes, and J. Horejsi, Fortschr. Phys. 42, 101 (1994).

[5] J. C. Collins, Acta Phys. Pol. B 34, 3103 (2003), https:// www.actaphys.uj.edu.pl/R/34/6/3103/pdf.

[6] X.-d. Ji, J.-p. Ma, and F. Yuan, Phys. Rev. D 71, 034005 (2005).

[7] J. C. Collins and D. E. Soper, Nucl. Phys. B194, 445 (1982).

[8] Y. L. Dokshitzer, Sov. Phys. JETP 46, 641 (1977), http:// www.jetp.ac.ru/cgi-bin/dn/e_046_04_0641.pdf.

[9] V. Gribov and L. Lipatov, Sov. J. Nucl. Phys. 15, 438 (1972).

[10] L. Lipatov, Sov. J. Nucl. Phys. 20, 94 (1975).

[11] G. Altarelli and G. Parisi, Nucl. Phys. B126, 298 (1977).

[12] A. Efremov and A. Radyushkin, Phys. Lett. 94B, 245 (1980).

[13] G. P. Lepage and S. J. Brodsky, Phys. Rev. D 22, 2157 (1980).

[14] X.-D. Ji, J. Phys. G 24, 1181 (1998).

[15] X.-D. Ji, Phys. Rev. D 55, 7114 (1997).

[16] M. Diehl, Phys. Rep. 388, 41 (2003).

[17] X. Ji, Annu. Rev. Nucl. Part. Sci. 54, 413 (2004).

[18] A. V. Belitsky and A. V. Radyushkin, Phys. Rep. 418, 1 (2005).

[19] K. Kumericki, S. Liuti, and H. Moutarde, Eur. Phys. J. A 52, 157 (2016).

[20] J. Collins, Cambridge Monogr. Part. Phys., Nucl. Phys., Cosmol. 32, 1 (2011), http://www.cambridge.org/de/ knowledge/isbn/item5756723.

[21] X. Ji, Phys. Rev. Lett. 110, 262002 (2013).

[22] X. Ji, Sci. China Phys. Mech. Astron. 57, 1407 (2014).

[23] X. Xiong, X. Ji, J.-H. Zhang, and Y. Zhao, Phys. Rev. D 90, 014051 (2014).

[24] H.-W. Lin, J.-W. Chen, S. D. Cohen, and X. Ji, Phys. Rev. D 91, 054510 (2015).

[25] C. Alexandrou, K. Cichy, V. Drach, E. Garcia-Ramos, K. Hadjiyiannakou, K. Jansen, F. Steffens, and C. Wiese, Phys. Rev. D 92, 014502 (2015).

[26] J.-W. Chen, S. D. Cohen, X. Ji, H.-W. Lin, and J.-H. Zhang, Nucl. Phys. B911, 246 (2016).

[27] C. Alexandrou, K. Cichy, M. Constantinou, K. Hadjiyiannakou, K. Jansen, F. Steffens, and C. Wiese, Phys. Rev. D 96, 014513 (2017).

[28] R. A. Briceño, M. T. Hansen, and C. J. Monahan, Phys. Rev. D 96, 014502 (2017).

[29] M. Constantinou and H. Panagopoulos, Phys. Rev. D 96, 054506 (2017). 
[30] C. Alexandrou, K. Cichy, M. Constantinou, K. Hadjiyiannakou, K. Jansen, H. Panagopoulos, and F. Steffens, Nucl. Phys. B923, 394 (2017).

[31] X. Ji, J.-H. Zhang, and Y. Zhao, Nucl. Phys. B924, 366 (2017).

[32] X. Ji, J.-H. Zhang, and Y. Zhao, Phys. Rev. Lett. 120, 112001 (2018).

[33] T. Ishikawa, Y.-Q. Ma, J.-W. Qiu, and S. Yoshida, Phys. Rev. D 96, 094019 (2017).

[34] J. Green, K. Jansen, and F. Steffens, Phys. Rev. Lett. 121, 022004 (2018).

[35] W. Wang, S. Zhao, and R. Zhu, Eur. Phys. J. C 78, 147 (2018).

[36] I. W. Stewart and Y. Zhao, Phys. Rev. D 97, 054512 (2018).

[37] T. Izubuchi, X. Ji, L. Jin, I. W. Stewart, and Y. Zhao, Phys. Rev. D 98, 056004 (2018).

[38] C. Alexandrou, K. Cichy, M. Constantinou, K. Jansen, A. Scapellato, and F. Steffens, Phys. Rev. Lett. 121, 112001 (2018).

[39] C. Alexandrou, K. Cichy, M. Constantinou, K. Jansen, A. Scapellato, and F. Steffens, Phys. Rev. D 98, 091503(R) (2018).

[40] J.-H. Zhang, J.-W. Chen, L. Jin, H.-W. Lin, A. Schäfer, and Y. Zhao, Phys. Rev. D 100, 034505 (2019).

[41] R. A. Briceño, J. V. Guerrero, M. T. Hansen, and C. J. Monahan, Phys. Rev. D 98, 014511 (2018).

[42] G. Spanoudes and H. Panagopoulos, Phys. Rev. D 98, 014509 (2018).

[43] Y.-S. Liu et al. (Lattice Parton Collaboration), Phys. Rev. D 101, 034020 (2020).

[44] A. Radyushkin, Phys. Lett. B 788, 380 (2019).

[45] J.-H. Zhang, X. Ji, A. Schäfer, W. Wang, and S. Zhao, Phys. Rev. Lett. 122, 142001 (2019).

[46] Z.-Y. Li, Y.-Q. Ma, and J.-W. Qiu, Phys. Rev. Lett. 122, 062002 (2019).

[47] C. Alexandrou, K. Cichy, M. Constantinou, K. Hadjiyiannakou, K. Jansen, A. Scapellato, and F. Steffens, Phys. Rev. D 99, 114504 (2019).

[48] W. Wang, J.-H. Zhang, S. Zhao, and R. Zhu, Phys. Rev. D 100, 074509 (2019).

[49] J.-W. Chen, H.-W. Lin, and J.-H. Zhang, Nucl. Phys. B952, 114940 (2020).

[50] T. Izubuchi, L. Jin, C. Kallidonis, N. Karthik, S. Mukherjee, P. Petreczky, C. Shugert, and S. Syritsyn, Phys. Rev. D 100, 034516 (2019).

[51] K. Cichy, L. Del Debbio, and T. Giani, J. High Energy Phys. 10 (2019) 137.

[52] W. Wang, Y.-M. Wang, J. Xu, and S. Zhao, Phys. Rev. D 102, 011502(R) (2020).

[53] H.-D. Son, A. Tandogan, and M. V. Polyakov, Phys. Lett. B 808, 135665 (2020).

[54] J. R. Green, K. Jansen, and F. Steffens, Phys. Rev. D 101, 074509 (2020).

[55] Y. Chai et al., Phys. Rev. D 102, 014508 (2020).

[56] V. Braun, K. Chetyrkin, and B. Kniehl, J. High Energy Phys. 07 (2020) 161

[57] S. Bhattacharya, K. Cichy, M. Constantinou, A. Metz, A. Scapellato, and F. Steffens, Phys. Rev. D 102, 111501(R) (2020).
[58] S. Bhattacharya, K. Cichy, M. Constantinou, A. Metz, A. Scapellato, and F. Steffens, Phys. Rev. D 102, 034005 (2020).

[59] S. Bhattacharya, K. Cichy, M. Constantinou, A. Metz, A. Scapellato, and F. Steffens, Phys. Rev. D 102, 114025 (2020).

[60] L.-B. Chen, W. Wang, and R. Zhu, Phys. Rev. D 102, 011503(R) (2020).

[61] L.-B. Chen, W. Wang, and R. Zhu, J. High Energy Phys. 10 (2020) 079.

[62] L.-B. Chen, W. Wang, and R. Zhu, arXiv:2006.14825.

[63] X. Ji, Y. Liu, A. Schäfer, W. Wang, Y.-B. Yang, J.-H. Zhang, and Y. Zhao, arXiv:2008.03886.

[64] K.-F. Liu and S.-J. Dong, Phys. Rev. Lett. 72, 1790 (1994).

[65] W. Detmold and C. J. David Lin, Phys. Rev. D 73, 014501 (2006).

[66] V. Braun and D. Mueller, Eur. Phys. J. C 55, 349 (2008).

[67] G. S. Bali et al., Eur. Phys. J. C 78, 217 (2018).

[68] G. S. Bali, V. M. Braun, B. Gläßle, M. Göckeler, M. Gruber, F. Hutzler, P. Korcyl, A. Schäfer, P. Wein, and J.-H. Zhang, Phys. Rev. D 98, 094507 (2018).

[69] W. Detmold, I. Kanamori, C. J. D. Lin, S. Mondal, and Y. Zhao, Proc. Sci., LATTICE2018 (2018) 106.

[70] J. Liang, T. Draper, K.-F. Liu, A. Rothkopf, and Y.-B. Yang (XQCD Collaboration), Phys. Rev. D 101, 114503 (2020).

[71] Y.-Q. Ma and J.-W. Qiu, Phys. Rev. D 98, 074021 (2018).

[72] Y.-Q. Ma and J.-W. Qiu, Int. J. Mod. Phys. Conf. Ser. 37, 1560041 (2015).

[73] A. Radyushkin, Phys. Lett. B 767, 314 (2017).

[74] A. J. Chambers, R. Horsley, Y. Nakamura, H. Perlt, P. E. L. Rakow, G. Schierholz, A. Schiller, K. Somfleth, R. D. Young, and J. M. Zanotti, Phys. Rev. Lett. 118, 242001 (2017).

[75] A. V. Radyushkin, Phys. Rev. D 96, 034025 (2017).

[76] K. Orginos, A. Radyushkin, J. Karpie, and S. Zafeiropoulos, Phys. Rev. D 96, 094503 (2017).

[77] Y.-Q. Ma and J.-W. Qiu, Phys. Rev. Lett. 120, 022003 (2018).

[78] A. V. Radyushkin, Phys. Lett. B 781, 433 (2018).

[79] A. Radyushkin, Phys. Rev. D 98, 014019 (2018).

[80] J.-H. Zhang, J.-W. Chen, and C. Monahan, Phys. Rev. D 97, 074508 (2018).

[81] J. Karpie, K. Orginos, and S. Zafeiropoulos, J. High Energy Phys. 11 (2018) 178.

[82] R. S. Sufian, J. Karpie, C. Egerer, K. Orginos, J.-W. Qiu, and D. G. Richards, Phys. Rev. D 99, 074507 (2019).

[83] B. Joó, J. Karpie, K. Orginos, A. Radyushkin, D. Richards, and S. Zafeiropoulos, J. High Energy Phys. 12 (2019) 081.

[84] A. V. Radyushkin, Phys. Rev. D 100, 116011 (2019).

[85] B. Joó, J. Karpie, K. Orginos, A. V. Radyushkin, D. G. Richards, R. S. Sufian, and S. Zafeiropoulos, Phys. Rev. D 100, 114512 (2019).

[86] I. Balitsky, W. Morris, and A. Radyushkin, Phys. Lett. B 808, 135621 (2020).

[87] A. Radyushkin, Int. J. Mod. Phys. A 35, 2030002 (2020).

[88] R. S. Sufian, C. Egerer, J. Karpie, R. G. Edwards, B. Joó, Y.-Q. Ma, K. Orginos, J.-W. Qiu, and D. G. Richards, Phys. Rev. D 102, 054508 (2020). 
[89] B. Joó, J. Karpie, K. Orginos, A. V. Radyushkin, D. G. Richards, and S. Zafeiropoulos, Phys. Rev. Lett. 125, 232003 (2020).

[90] M. Bhat, K. Cichy, M. Constantinou, and A. Scapellato, arXiv:2005.02102.

[91] K. Can et al., Phys. Rev. D 102, 114505 (2020).

[92] C. Alexandrou, M. Constantinou, K. Hadjiyiannakou, K. Jansen, and F. Manigrasso, arXiv:2009.13061.

[93] J. Bringewatt, N. Sato, W. Melnitchouk, J.-W. Qiu, F. Steffens, and M. Constantinou, Phys. Rev. D [arXiv:2010.00548].

[94] K. Cichy and M. Constantinou, Adv. High Energy Phys. 2019, 3036904 (2019).

[95] X. Ji, Y.-S. Liu, Y. Liu, J.-H. Zhang, and Y. Zhao, arXiv:2004.03543.

[96] M. Constantinou, in 38th International Symposium on Lattice Field Theory, arXiv:2010.02445.

[97] C. Alexandrou, K. Cichy, M. Constantinou, K. Hadjiyiannakou, K. Jansen, A. Scapellato, and F. Steffens, Proc. Sci., LATTICE2019 (2019) 036 [arXiv:1910.13229].

[98] S. Bhattacharya, C. Cocuzza, and A. Metz, Phys. Lett. B 788, 453 (2019).

[99] S. Bhattacharya, C. Cocuzza, and A. Metz, Phys. Rev. D 102, 054021 (2020).

[100] The operator $\gamma_{3}$ (unpolarized) is no longer used as it mixes with a twist-3 distribution [29].

[101] For an alternative prescription see Ref. [34].

[102] G. Martinelli, C. Pittori, C. T. Sachrajda, M. Testa, and A. Vladikas, Nucl. Phys. B445, 81 (1995).

[103] See Supplemental Material at http://link.aps.org/supplemental/ 10.1103/PhysRevLett.125.262001 for a description of the methodology, technical aspects and additional figures.

[104] Y.-S. Liu, W. Wang, J. Xu, Q.-A. Zhang, J.-H. Zhang, S. Zhao, and Y. Zhao, Phys. Rev. D 100, 034006 (2019).

[105] For older work on the matching of quasi-GPDs in the transverse momentum cutoff scheme, see Refs. [128,129].

[106] G. Backus and F. Gilbert, Geophys. J. Int. 16, 169 (1968).

[107] R. Frezzotti, P. A. Grassi, S. Sint, and P. Weisz (Alpha Collaboration), J. High Energy Phys. 08 (2001) 058.

[108] R. Frezzotti and G. C. Rossi, J. High Energy Phys. 08 (2004) 007.
[109] B. Sheikholeslami and R. Wohlert, Nucl. Phys. B259, 572 (1985).

[110] C. Alexandrou et al., Phys. Rev. D 98, 054518 (2018).

[111] G. S. Bali, B. Lang, B. U. Musch, and A. Schäfer, Phys. Rev. D 93, 094515 (2016).

[112] C. Morningstar and M. J. Peardon, Phys. Rev. D 69, 054501 (2004).

[113] C. Alexandrou, M. Constantinou, K. Hadjiyiannakou, K. Jansen, H. Panagopoulos, and C. Wiese, Phys. Rev. D 96, 054503 (2017).

[114] C. Alexandrou, S. Bacchio, M. Constantinou, J. Finkenrath, K. Hadjiyiannakou, K. Jansen, G. Koutsou, H. Panagopoulos, and G. Spanoudes, Phys. Rev. D 101, 094513 (2020).

[115] M. Albanese et al. (APE Collaboration), Phys. Lett. B 192, 163 (1987).

[116] S. Gusken, Nucl. Phys. B, Proc. Suppl. 17, 361 (1990).

[117] C. Alexandrou, S. Gusken, F. Jegerlehner, K. Schilling, and R. Sommer, Nucl. Phys. B414, 815 (1994).

[118] M. Gockeler, R. Horsley, H. Oelrich, H. Perlt, D. Petters, P. E. L. Rakow, A. Schafer, G. Schierholz, and A. Schiller, Nucl. Phys. B544, 699 (1999).

[119] C. Alexandrou, M. Constantinou, and H. Panagopoulos (ETM Collaboration), Phys. Rev. D 95, 034505 (2017).

[120] M. Constantinou et al. (ETM Collaboration), J. High Energy Phys. 08 (2010) 068.

[121] A. N. Tikhonov, Sov. Math. Dokl. 4, 1035 (1963).

[122] M. V. Ulybyshev, C. Winterowd, and S. Zafeiropoulos, EPJ Web Conf. 175, 03008 (2018).

[123] M. Ulybyshev, C. Winterowd, and S. Zafeiropoulos, Phys. Rev. B 96, 205115 (2017).

[124] C. Alexandrou, M. Constantinou, S. Dinter, V. Drach, K. Jansen, C. Kallidonis, and G. Koutsou, Phys. Rev. D 88, 014509 (2013).

[125] F. Yuan, Phys. Rev. D 69, 051501(R) (2004).

[126] A. Biselli (CLAS Collaboration), J. Phys. Conf. Ser. 938, 012003 (2017).

[127] A. Accardi et al., Eur. Phys. J. A 52, 268 (2016).

[128] X. Ji, A. Schäfer, X. Xiong, and J.-H. Zhang, Phys. Rev. D 92, 014039 (2015).

[129] X. Xiong and J.-H. Zhang, Phys. Rev. D 92, 054037 (2015). 